\title{
Acute Gangrenous Acalculous Cholecystitis In An Adolescent: A Case Report
}

\author{
Dr Hemendra Kumar, Dr Ankit Jain, Dr Dhiraj Sharma, Dr Md. Jawed Akther \\ People's College Of Medical Science And Research Center, Bhopal, India
}

\begin{abstract}
Introduction: Acute Acalculous cholecystitis (AAC) represents a severe complicated variant of acute gallbladder $(G B)$ disease. AAC most often occurs in old age, critically ill patients, especially related to trauma, surgery, shock, burns, sepsis, total parenteral nutrition, diabetes, immunosupression and/or prolonged fasting. The presentation of acalculous cholecystitis is similar to that of calculous disease.

Case Report: An 18 year old male patient presented with severe abdominal pain associated with tenderness and guarding in right hypochondrium and epigastric region. Radiological investigations were suggestive of acute acalculous cholecystitis. Cholecystectomy was performed subsequently.

Discussion: $A A C$ is defined as an acute necroinflammatory disease of the gallbladder in the absence of cholelithiasis. The etiology of AAC is multifactorial and likely results from bile stasis or ischemia or both. Ultrasonography (USG) is considered as first modality to evaluate suspected AAC and diagnostic triad is GB wall thickness, sludge, and hydrops. Computed Tomography (CT) scan can be performed if other abdominal pathology is considered more likely. Treatment options for AAC are cholecystostomy and cholecystectomy. Cholecystectomy generally is considered the definitive therapy. Cholecystostomy can be a lifesaving alternative in the patient considered too unstable to undergo definitive surgery.

Conclusion: The possibility of AAC, especially in a younger patient with symptoms of acute cholecystitis should always be considered, in spite of the absence of any medical co morbid condition and risk factors. Proper clinical assessment and interpretation of investigation with high index of suspicion with timely intervention can reduce mortality and morbidity associated with AAC.
\end{abstract}

Key word: Acute Acalculous cholecystitis (AAC), Adolescent, risk factor.

\section{Introduction}

Acute Acalculous cholecystitis (AAC) represents a severe complicated variant of acute gallbladder (GB) disease. AAC most often occurs in old age, critically ill patients, especially related to trauma, surgery, shock, burns, sepsis, total parenteral nutrition, diabetes, immunosupression and/or prolonged fasting. The presentation of acalculous cholecystitis is similar to that of calculous disease. The disease process is generally more fulminant than that of calculous cholecystitis and may progress to gangrene and perforation of the gallbladder leading to high mortality and morbidity. Early diagnosis can be made by high index of suspicion with proper interpretation of clinical finding and investigations to decrease both mortality and morbidity ${ }^{1,2}$. We are presenting here a rare case of acute gangrenous acalculus cholecystitis in an adolescent patient without any medical co morbid condition and risk factor.

\section{Case Report}

An 18 year old male patient came to our emergency department at, People's College of Medical Science and Research Center, Bhopal, India, a teaching hospital with complain of abdominal pain which was severe, diffuse and continuous in nature, of one day duration. It was associated with multiple episodes of vomiting. There was no similar previous history or history of hospitalization and trauma.

On physical examination patient was afebrile with tachycardia \& tachypnea. Abdominal examination revealed marked tenderness in right hypochondrium and epigastric region with diffuse guarding all over abdomen. On auscultation bowel sound was sluggish.

Laboratory investigations on admission revealed marked leukocytosis $\left(21.4 \times 10^{9} / \mathrm{L}\right)$, and mildly raised liver enzymes. Plain radiograph of abdomen was insignificant. Abdominal Ultrasonography (USG) revealed a distended gallbladder with minimal fluid collection in perihepatic region and mild distention of large bowel.

Patient was managed in the line of acute cholecystitis. But because of unsatisfactory response to treatment except decreasing leucocyte count, Contrast enhanced computed tomography scan (CECT) of abdomen was advised. CECT abdomen revealed distended gall bladder with thickened and irregular wall. No intra-hepatic or extra-hepatic duct dilatation was seen. There was a thin rim of fluid anterior to the surface of the liver and between gallbladder and duodenum, suggestive of acute acalculous cholecystitis with reactionary fluid 
around gallbladder (Fig 1). Also rectum, sigmoid colon and ascending colon appeared dilated. USG guided tapping revealed frank intra peritoneal bile, suggesting it to be gallbladder perforation.

Intra-operatively, Approximately1000 $\mathrm{ml}$ of bilious fluid was aspirated from peritoneal cavity (Fig 2) confined to right upper quadrant. The gallbladder was having multiple gangrenous patches with subserosal grayish fluid over the fundal area. Gall bladder was not adherent to omentum or any other structure. Cholecystectomy was performed followed by thorough lavage of peritoneal cavity. On opening the gallbladder, there were multiple patchy areas with sloughed out mucosa, and bile with debris was present without any calculous (Fig 3). Liver, Stomach, all parts of small intestine and large intestine were healthy. Post operative Widal test was not in favour of Typhoid, excluding it as common cause for gangrenous acalculous cholecystitis in young age.

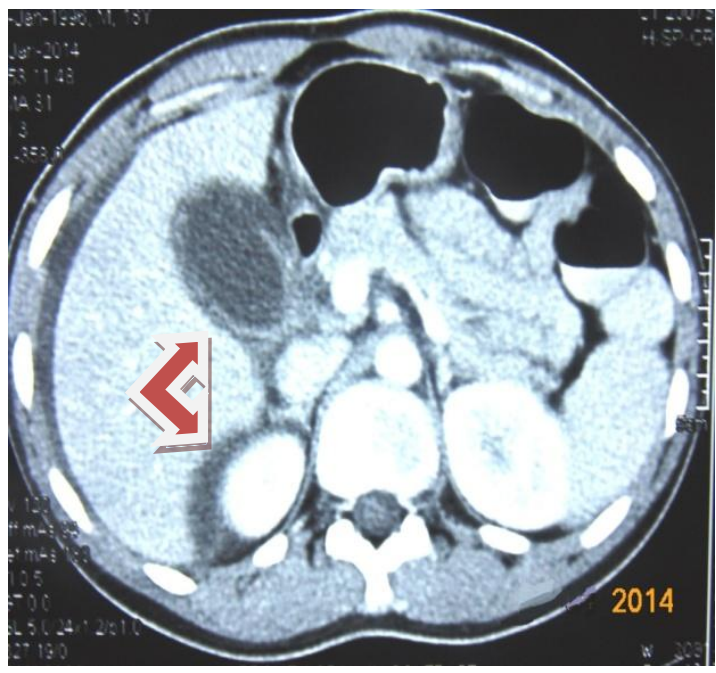

Fig.1 distended GB, perihepatic \& sub-hepatic collection

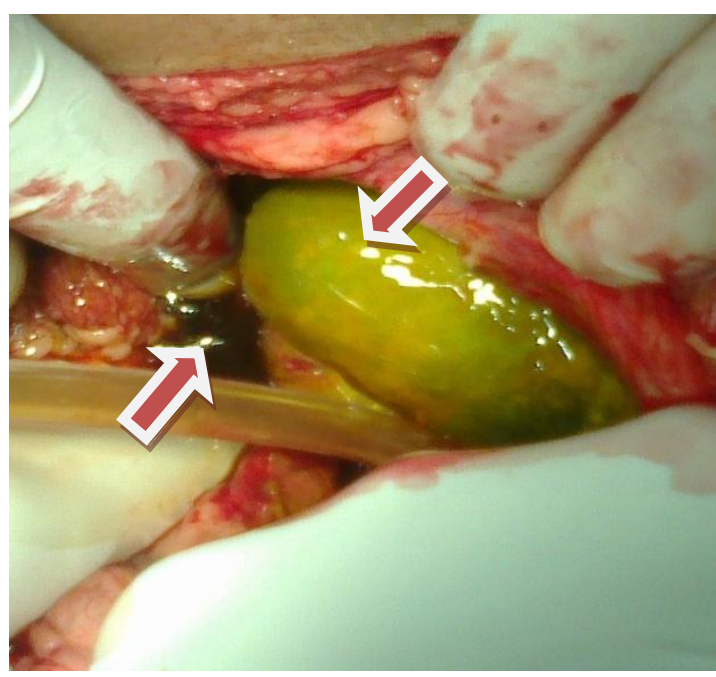

Fig.2 bilious fluid \& Gangrenous GB

Multiple sections of tissue from fundus \& body of gallbladder on histopathological examination (HPE) revealed mostly transmural destruction \& necrosis of the wall. There was diffuse infiltration comprising of mostly neutrophil, pus cell with focal area of infiltration by chronic inflammatory cell like lymphocyte \& monocyte, areas of hemorrhage, congested blood vessels \& bile collection at places in supra mucosal areas (Fig 4).

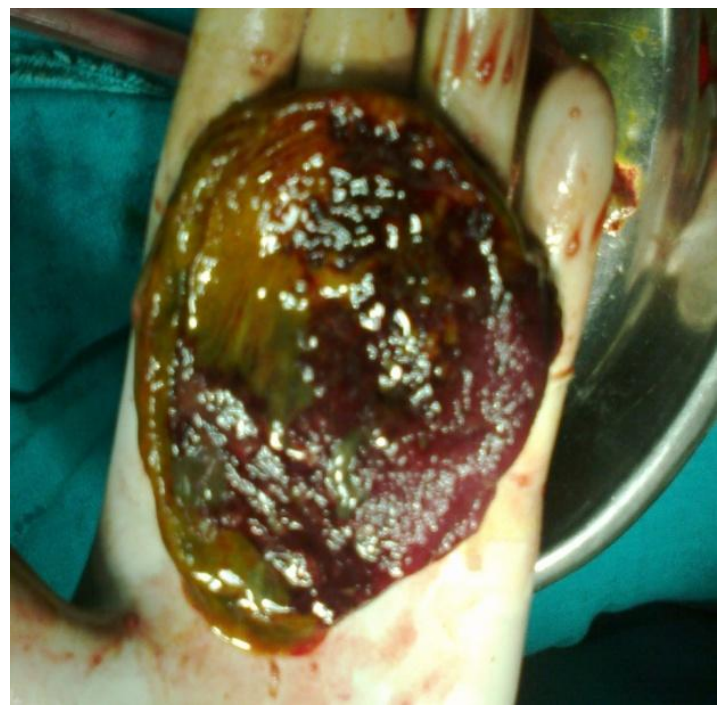

Fig.3 GB with multiple gangrenous patches

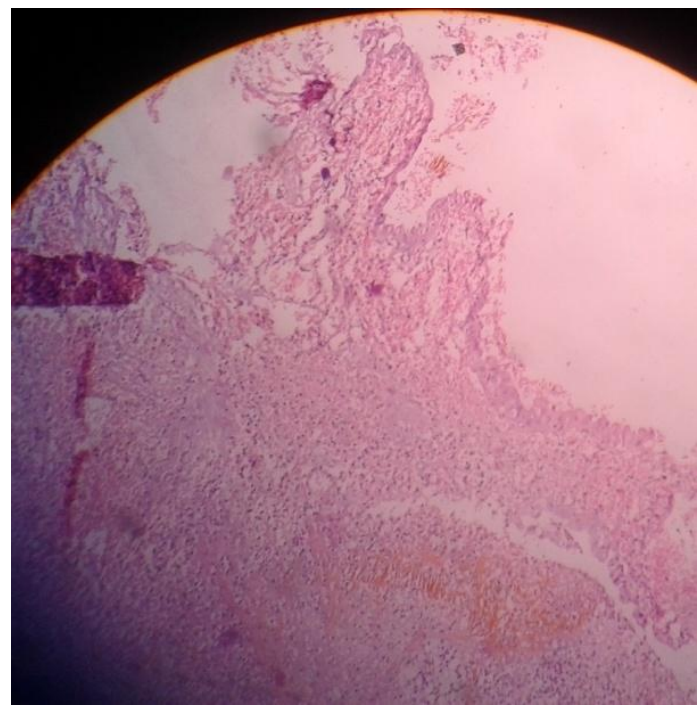

Fig.4 HPE slide of GB 
Post-operatively patient improved dramatically with normalization of tachycardia and leukocyte count on next day. Patient was started with oral feed on $2^{\text {nd }}$ post operative day and discharged uneventfully on seventh day after the operation.

\section{Discussion}

AAC is defined as an acute necroinflammatory disease of the gallbladder in the absence of cholelithiasis and has a multifactorial pathogenesis. ${ }^{3,4}$ It accounts for approximately $10 \%$ (range, $2 \%-15 \%$ ) of all cases of acute cholecystitis. It occurs in about $0.2 \%$ to $0.4 \%$ of all critically ill old patients usually about 60 years of age, with an approximate male: female ratio of 2 to $3: 1$. $^{4,5}$

Clinically, AAC is indistinguishable from acute calculous cholecystitis ${ }^{10,11}$ as clinical findings like right upper-quadrant pain, fever, leukocytosis, and abnormal liver tests are nonspecific to it. ${ }^{3,4,6}$

AAC can progress to gangrene, perforation, and empyema of gall bladder. These complications occur in $6 \%$ to $82 \%$ of cases. ${ }^{3,7,8}$ Most studies had revealed high mortality rate associated with AAC ( $10 \%-90 \%$ with early or late diagnosis, respectively ${ }^{3,5,8,9}$ ).

The etiology of AAC is multifactorial and likely results from bile stasis or ischemia or both. Bile stasis can be caused by fasting, obstruction, postsurgical/procedural irritation or ileus (total parenteral nutrition [TPN]), which can lead to bile inspissation that is directly toxic to the gallbladder epithelium. ${ }^{10,11}$ Laurila et al ${ }^{5}$ provided excellent histologic data that explain some of the pathology of AAC as a response to systemic inflammation. AAC showed the following: (1) increased leukocyte margination (corresponding to ischemia and reperfusion injury $)^{12}$; (2) increased focal lymphatic dilation with interstitial edema associated with local microvascular occlusion (ischemia related); and (3) increased and deeper bile infiltration in the GB wall suggesting that bile stasis and increased epithelial permeability exist, leading to epithelial damage. These findings corroborate the hypotheses that bile stasis and ischemia likely are involved in the pathogenesis of AAC. Typhoid fever is also known to occasionally cause spontaneous perforation of the gall bladder in the absence of stone disease in young $\operatorname{age}^{13}$.It is evident that despite many attempts to elucidate the pathogenesis of AAC, it is still not completely defined.

The diagnosis of AAC is difficult because no clinical findings establish it. ${ }^{3,4,6,8}$

USG is considered as first modality to evaluate suspected AAC. The most studied diagnostic criteria for USG are GB wall thickness, pericholecystic fluid, subserosal edema, intramural gas, sloughed mucosa, sludge, and hydrops. ${ }^{14,15}$ The most studied and cited criteria the so-called diagnostic triad is GB wall thickness, sludge, and hydrops. ${ }^{3,14,15}$ The sensitivity and specificity of USG to diagnose AAC range from $30 \%$ to $100 \%$. Computed tomography (CT) is as accurate as ultrasound in the diagnosis of AAC.${ }^{14} \mathrm{CT}$ diagnostic criteria are similar to USG. ${ }^{16}$. Preference may be given to $\mathrm{CT}$ if other abdominal pathology is considered more likely.

The two prevailing treatment options for AAC are cholecystostomy (drainage of the GB) and cholecystectomy. Cholecystectomy is generally considered definitive therapy if it can be performed, ${ }^{2,3,8,11}$ and some aggressively perform only open cholecystectomies. Laparoscopic cholecystectomy can also be performed, though may require conversion to open procedure. ${ }^{5}$ Cholecystostomy can be a lifesaving alternative in the patient considered too unstable to undergo definitive procedure ${ }^{17}$, but provides inadequate drainage of the common bile duct for concomitant cholangitis. Cholecystostomy may provide time to optimize the patient's condition for surgery. ${ }^{17}$ and therefore it is considered by some as a bridge to safer cholecystectomy. Laurila et $\mathrm{a}^{5}$ aggressively favor open cholecystectomy, even in the critically ill, to reverse or prevent multiorgan failure.

Our patient had complicated AAC with gangrene and perforation of GB. We presumed that there might be small rent in the gangrenous area of the gallbladder which was sealed off by firm adherence of debris. This could be the possible reason for gallbladder distention with biliary peritonitis in our case.

\section{Conclusion}

Acute Acalculous Cholecystitis is difficult to diagnose, but an early correct clinical assessment, proper interpretation of laboratory and radiological investigations, and high index of suspicion is essential for successful diagnosis and treatment, which is readily available. The possibility of AAC, especially in a younger patient with symptoms of acute cholecystitis should always be considered, in spite of the absence of any medical co morbid condition and risk factors. Timely intervention can reduce mortality and morbidity associated with AAC.

\section{Consent}

Written informed consent was obtained from the legal guardian of the patient for publication of this case report and any accompanying images. A scaned copy of the written consent is available for review by the Editor-in- Chief of this journal. 


\section{Acknowledgement}

I thank Dr. Md. Jawed Akther, Associate Professor, Surgery who was chief of our operative team. I also thank Dr. R. S. Hajari, Professor, Pathology for review of pathological material.

\section{References}

[1]. Jason L. Huffman,Steven Schenkar; Acute Acalculous Cholecystitis: A Review; cghjournal 2010;8(1):15-22.

[2]. Patrick G, Jackson, Steven R.T. Evans. Biliary system. In Townsand, editor. Sabiston Text Book of Surgery, $19^{\text {th }}$ ed. Elsevier Publishers; 2012.p.1501-02.

[3]. Barie PS, Eachempati SR. Acute acalculous cholecystitis. Curr Gastroenterol Rep 2003; 5:302-309.

[4]. Owen CC, Jain R. Acute acalculous cholecystitis. Curr Treat Options Gastroenterol 2005; 8:99-104.

[5]. Laurila J, Laurila PA, Saarnio J, et al. Organ system dysfunction following open cholecystectomy for acute acalculous cholecystitis in critically ill patients. Acta Anaesthesiol Scand 2006; 50: 173-179.

[6]. Babb RR. Acute acalculous cholecystitis. A review. J Clin Gastroenterol 1992; 15:238-241.

[7]. Iaria C, Arena L, Di Maio G, et al. Acute acalculous cholecystitis during the course of primary Epstein-Barr virus infection: a new case and a review of the literature. Int J Infect Dis 2008; 12:391-395.

[8]. 8.Wang AJ, Wang TE, Lin CC, et al. Clinical predictors of severe gallbladder complications in acute acalculous cholecystitis. World J Gastroenterol 2003; 9:2821-2823.

[9]. 9.Laurila JJ, Ala-Kokko TI, Laurila PA, et al. Histopathology of acute acalculous cholecystitis in critically ill patients. Histopathology 2005; 47:485-492

[10]. 10. McChesney JA, Northup PG, Bickston SJ. Acute acalculous cholecystitis associated with systemic sepsis and visceral arterial hypoperfusion: a case series and review of pathophysiology. Dig Dis Sci 2003; 48:1960-1967.

[11]. 11. Shuman WP, Rogers JV, Rudd TG, et al. Low sensitivity of sonography and cholescintigraphy in acalculous cholecystitis. AJR Am J Roentgenol 1984; 142:531-534.

[12]. 12. Taoka H. Experimental study on the pathogenesis of acute acalculous cholecystitis, with special reference to the roles of microcirculatory disturbances, free radicals and membrane-bound phospholipase A2. Gastroenterol Jpn 1991; 26:633-644.

[13]. 13.Gnassingbé K, Katakoa G, Kanassoua KK, Adabra K, Mama WA, Simlawo K, Eteh K, Tekou H. Acute cholecystitis from typhic origin in children. Afr J Paediatr Surg 2013; 10:108-11

[14]. 14. Mirvis SE, Vainright JR, Nelson AW, et al. The diagnosis of acute acalculous cholecystitis: a comparison of sonography, scintigraphy, and CT. AJR Am J Roentgenol 1986; 147:1171-1175.

[15]. 15. Boland GWL, Slater G, Lu DSK, et al. Prevalence and significance of gallbladder abnormalities seen on sonography in intensive care unit patients. AJR Am J Roentgenol 2000; 174:973-977.

[16]. 16. Mirvis SE, Whitley NO, Miller JW. CT diagnosis of acalculous cholecystitis. J Comput Assist Tomogr 1987; 11:83-87.

[17]. 17. Glenn F. Cholecystostomy in the high-risk patient with biliary tract disease. Ann Surg. (1977);185:185-191. [PMC free article: PMC1396112] [PubMed: 836091 